\title{
Creating Interactive Scientific Publications using Bindings
}

\author{
MARKUS KONKOL, University of Münster, Institute for Geoinformatics, Germany \\ CHRISTIAN KRAY, University of Münster, Institute for Geoinformatics, Germany \\ JAN SULEIMAN, University of Münster, Institute for Geoinformatics, Germany
}

\begin{abstract}
Many scientific publications report on computational results based on code and data, but even when code and data are published, the main text is usually provided in a separate, traditional format such as PDF. Since code, data, and text are not linked on a deep level, it is difficult for readers and reviewers to understand and retrace how the authors achieved a specific result that is reported in the main text, e.g. a figure, table, or number. In addition, to make use of new the opportunities afforded by data and code availability, such as re-running analyses with changed parameters, considerable effort is required. In order to overcome this issue and to enable more interactive publications that support scientists in more deeply exploring the reported results, we present the concept, implementation, and initial evaluation of bindings. A binding describes which data subsets, code lines, and parameters produce a specific result that is reported in the main text (e.g. as a figure, table, or number). Based on a prototypical implementation of these bindings, we propose a toolkit for authors to easily create interactive figures by connecting specific UI widgets (e.g. a slider) to parameters. In addition to inspecting code and data, readers can then manipulate the parameter and see how the results change. We evaluated the approach by applying it to a set of existing articles. The results provide initial evidence that the concept is feasible and applicable to many papers with moderate effort.
\end{abstract}

CCS Concepts: • Human-centered computing $\rightarrow$ Interactive systems and tools;

Additional Key Words and Phrases: open science, reproducible research, research compendia, interactive papers

ACM Reference Format:

Markus Konkol, Christian Kray, and Jan Suleiman. 2019. Creating Interactive Scientific Publications using Bindings. Proc. ACM Hum.-Comput. Interact. 3, EICS, Article 16 (June 2019), 18 pages. https://doi.org/10.1145/ 3331158

\section{INTRODUCTION}

Many scientific articles include computational results, such as figures, tables, and numbers, that are based on source code and data. For this reason, the open science initiatives "open code" and "open data" request authors to publish the materials underlying these results. Readers and reviewers should be able to understand how the authors produced the computational results, which parameters were used for the analysis, and how manipulations to these parameters affect the results. Reviewers and readers could thus check whether the results vary after changing the original parameter set and reuse the materials for their own research. Increasingly, journals and funding agencies are instituting guidelines to ensure that researchers share their materials.

Authors' addresses: Markus Konkol, m.konkol@uni-muenster.de, University of Münster, Institute for Geoinformatics, Heisenbergstrasse 2, 48149 Münster, NRW, Münster, Germany; Christian Kray, c.kray@uni-muenster.de, University of Münster, Institute for Geoinformatics, Heisenbergstrasse 2, 48149 Münster, NRW, Münster, Germany; Jan Suleiman, j_sule02@uni-muenster.de, University of Münster, Institute for Geoinformatics, Heisenbergstrasse 2, 48149 Münster, NRW, Münster, Germany.

This work was supported by the German Research Foundation (DFG) [grant number KR 3930/3-1].

Permission to make digital or hard copies of part or all of this work for personal or classroom use is granted without fee provided that copies are not made or distributed for profit or commercial advantage and that copies bear this notice and the full citation on the first page. Copyrights for third-party components of this work must be honored. For all other uses, contact the owner/author(s). (c) 2019 Copyright held by the owner/author(s).

2573-0142/2019/6-ART16

https://doi.org/10.1145/3331158

Proc. ACM Hum.-Comput. Interact., Vol. 3, No. EICS, Article 16. Publication date: June 2019. 
However, the way research is published has not yet adapted to these advancements. Scientific insights are still published as static texts, i.e. in PDF documents or HTML renderings. These published papers only provide links to supplemental materials and are thus largely disconnected from the underlying code and data. Consequently, gaining a deeper understanding of the analysis presented in the paper requires extensive effort, including downloading the supplements, opening the code file in the local environment (if installed), and searching for the right source code lines and data subsets. In addition, it is challenging to find out which parameters underlie a statistical analysis and how changes to their initial configuration affect the final output. Hence, readers might not even try to obtain a detailed understanding of the code used to produce the results. One way to simplify the inspection of the underlying materials and the manipulation of parameters would be to create interactive papers with the help of user interface (UI) widgets. UI widgets, such as a slider or radio buttons, are interactive elements which provide readers with some control over what is shown, for example, in an interactive figure [40]. However, such figures are rarely part of articles, as creating them is time-consuming.

The key contributions of this work are threefold: First, we present the concept of bindings in the context of scientific publications. The goal of a binding is to assist readers and reviewers in inspecting the code and the data underlying a specific result in the paper, in manipulating the underlying parameters to see how the output changes, and in substituting the dataset underlying the analysis. In order to achieve those goals, a binding describes which source code lines and data subsets were used to produce an individual computational result, such as a figure, table, or number in the text. This fine-grained link between code, data, and a specific result down to the level of parameters can help to make computational results more transparent. One of the main differences to related concepts (e.g. plotly [42]) is that bindings explicitly refer to single parameters which influence the result. A binding can then be used to create an interactive figure by connecting a UI widget to a parameter. Second, we describe an initial prototypical web application for authors to create a binding, and for readers to use the resulting interactive figure. The system assists authors in selecting the code lines, specifying the parameter, and configuring the UI widget. Based on the resulting binding, the system creates an interactive figure for readers, who can then use it in parallel to reading the text. Third, we showcase the feasibility of a binding by applying it to 83 reproducible figures in 20 existing scientific publications. The results show that bindings are applicable to scientific articles, because many of the figures in the papers are produced by computational analyses including parameters. We successfully created 83 interactive figures using a slider or radio buttons which manipulate the initial configuration of the parameters and thus the final output. We will also provide an initial assessment of the effort required to create a binding and the time needed to use the interactive figure.

Scope: The presented approach addresses papers which include computational results in the form of statistics or analyses. We thus do not consider papers describing conceptual results, frameworks, information systems, photos, or diagrams based on ready-to-use software. Due to the focus on statistics and to keep the scope of the work manageable, we only consider computational results based on $R$, a programming language for statistics [38]. Bindings require reproducible research results, which means that we can use the same data and code to achieve the same results as reported in the original paper [20].

In the remainder of this article, we first review related work and then present the concept of a binding followed by its prototypical implementation. Afterwards, we describe the evaluation and discuss the results. Finally, we conclude by summarising key insights and future work directions. 


\section{RELATED WORK}

In the following sections, we review work on open science practices, how research materials can be connected to increase transparency, and possibilities for creating interactive papers.

\subsection{Publishing research materials}

The publication of research materials, such as source code and data, is a key aspect in open science. Open science summarises initiatives that aim at improving the accessibility, reproducibility, replicability, and understandability of the scientific results reported in an article. These initiatives address individual aspects, for example, publishing "open access" articles [22], releasing "open data" [17], providing access to "open code" [13], and using "open source software" [44]. Yet, for a number of reasons (e.g. effort), authors are reluctant to publish all research materials [5]. Several studies from different domains have checked the accessibility of materials underlying certain computations and had poor results, for example, because code and data were missing (see, for example, Nüst et al. [31] in GIScience, Echtler and Häußler [14] in HCI). Several tools and concepts exist which try to minimise the effort authors have to put forward to publish all their materials, e.g. source code and data, in an open way. A basic concept is the research compendium [16], which is essentially a project folder containing all materials that are needed to recompute the scientific results. Online applications, such as Open Science Framework ${ }^{1}$ provide the infrastructure to realise this form of publishing research. In addition, the OntoSoft ontology describes metadata for research software in order to facilitate the reuse and sharing of existing software [18]. Nevertheless, access to these materials does not guarantee reproducibility of the computational results, e.g. the figures, tables, and numbers [45]. Technical issues might impede code execution, and the reproduced figures might be different from the original ones [28]. For this reason, several other approaches focus on executable environments: A Sciunit, for example, is a reusable research object allowing recomputation of the analysis in a container [15] (see also ReproZip [7]). A similar approach is the Executable Research Compendium (ERC) by Nüst et al. [32] which also considers the publication workflow of a scientific article. In order to demonstrate the potential of ERCs, Konkol and Kray [27] suggested and implemented an extended workflow for readers while examining a scientific article that was submitted as an ERC. The workflow contains the steps "discovery", i.e. finding relevant literature based on information included in the source code (e.g. a library or a function), "inspection", i.e. in-depth examination of code and data underlying the computational results, "manipulation", i.e. changing the parameters used in the analysis, and "substitution", i.e. replacing the used dataset with another compatible one. The realisation of these steps requires more than just accessible and reproducible source code, but it also requires fine-grained connections between text, data, and code down to the level of parameters.

\subsection{Connecting paper, code, and data}

The approaches presented above strongly focus on accessibility and reproducibility. However, the paper, the code, and the data are still disconnected, making it difficult for readers to relate the results reported in the paper to the source code and the data subsets [37]. Several approaches try to address this issues by connecting the research components. One approach is called "literate programming", a practice to mix text and code in one dynamic document [26]. Several systems build on this concept: fupyter notebook combines code and text in one interactive document [25]; $R M a r k d o w n$ combines markdown, i.e. plain text, and $R$ code which can be rendered to PDF files using journal templates [2]; and Sweave integrates text written in $L a T e X$ and $R$ code [29]. These documents can be easily shared between collaborators or attached to the paper. A similar approach

${ }^{1}$ Open science framework: https://osf.io/, last access: 21.02 .2019 
is StatTag [51]. Authors can mark code subsets underlying a specific result in the text, e.g. a number, table, or figure. The resulting linkage enables updates of the result in the text upon changes to the code. StatTag rather addresses authors who would like to work with a dynamic document, but to a lesser extent readers who might want to interact with the results while studying the paper.

In contrast to these solutions, other approaches treat text, data, and code as separate but linkable components. Creating such links can support the understanding of, for example, reviewers who verify the reported results and readers who reuse existing materials [3]. Kauppinen and de Espindola suggest "Linked open data" to connect data, code, and results [24]. Drees et al. [12] propose using permanent identifiers to connect the paper, data, software, and videos, thereby meeting the "FAIR" 2 principles [52]. Still, an extensive workload is needed to understand the source code, which is why Bacchelli et al. looked at email correspondences among software developers and users [4] Instead of linking the text to the entire code, they connect code-specific expressions in emails (e.g. classes, functionalities) to the particular section in the source code file. A similar approach was presented by Antoniol et al. who created fine-grained links between software documentations and the corresponding function and variables [3]. However, scientific articles are fundamentally different from emails or software documentations. Functions in the code are usually not explicitly mentioned in the paper, except they are the object of research.

To address this issue, Pham et al. [37] developed Sole. Authors can connect a result in the article produced by code to the corresponding code snippet by wrapping the relevant functions into tags. The system SciSoft X offers a similar functionality but also provides a UI for readers to examine the selected code and text in parallel [23]. To achieve that, authors create links between code snippets and the paragraph, which are then highlighted in the manuscript. Nevertheless, while Sole and SciSoftX support exploration and understanding of how results were achieved, they lack features which enable manipulation of parameters and reuse of executable code subsets. The approaches presented so far, i.e. research compendia, dynamic documents, and linked materials, pave the way for authors to go beyond the traditional way of publishing research as static PDF files. Interactive and executable papers promise to make research results more easily accessible, to help with understanding them (e.g. using interactive figures), and to facilitate reproduction of computational results.

\subsection{Creating interactive papers}

Increasingly, papers are being published as HTML renderings instead of traditional PDF files. Readers can study the paper as usual but also interact with it in a limited way, e.g. jump to references or download figures. Still, the scientific figures in these papers are usually static, although promising interaction possibilities exist, for example, to enable data exploration [35]. This is a meaningful approach, because readers can achieve a better understanding of computational results if they are given some control over what is shown in the figure $[1,50]$. In addition, interactive figures are also desirable for authors because, according to Greis et al. [21], authors' analyses of their data might be based on an inaccurate implementation of the model or on uncertain data due to, for example, imprecise measurements. For this reason, they suggest attaching sliders to figures which allow users to define a range as input.

Victor designed the Website worrydream, which allows readers to change the assumptions, i.e. the initial configuration of the parameters, underlying the results in the text [49] (see also the work by Dragicevic et al. [11]). Based on those changes, texts and figures adapt accordingly. Using interactive figures to convey results is not new but is rarely done in scientific articles [35]. To try to address this, the publisher Faculty of 1000 introduced the term "Living Figures" [43]; they published

${ }^{2}$ Research data should be findable, accessible, interoperable, and reusable. 
scientific articles including interactive figures using Plotly [42], a tool allowing readers to pan and zoom into diagrams to see exact values, and it incorporates UI widgets, such as a slider (for an example, see Colomb and Brembs [8], and Delory et al. [9]). However, creating these interactive elements is still a time-consuming task and done by journal staff or in the context of experiments Creating interactive figures is also possible with "literate programming" approaches. With few lines of code, users can attach UI widgets to figures embedded in fupyter notebooks using ipywidgets [30], or in RMarkdown documents using Shiny [6] and htmlwidgets [48]. Tools such as Vega lite [53] allow authors to provide links to external interactive figures.

On a rather programmatic level, tools such as Plumber [47] and OpenCPU [33] allow users to create APIs out of source code. Thus, common API requests (GET/POST) can be created to rerun the code with a different parameter value. Software engineers gain full control of how to enable users to manipulate a parameter and how to visualise the returned outcome (e.g. a figure).

In summary, several conceptual and technical solutions support authors in publishing open research. However, even if the computational results are accessible and reproducible, the analysis might not be transparent [19]. The code and data can still be complex, making it difficult to understand how an individual result, e.g. a specific figure, was computed, which parameters were used, and how changes to these affect the outcome. The concept of a binding as proposed in this work addresses this issue by creating fine-grained linkages between those code lines and data subsets that are needed to compute an individual result. A key difference to the approaches mentioned above is that bindings contain information about single parameters that substantially influence the specified result. We can use this information to create interactive papers which allow readers to manipulate the initial parameter values used to produce a figure, for example, by using a slider or radio buttons.

\section{BINDING}

In order to facilitate access to the research components underlying the computational results in a scientific article, we propose the concept of a binding. In the following subsections, we first provide a rationale for the design and then describe the key components of bindings.

\subsection{Design rationale}

The development process was informed by the needs of several stakeholders who were mentioned in [32]: authors and readers; publishers and libraries; and software developers. Authors and readers want to describe and inspect the connections between results, data, and analysis. In order to enable this, a binding has to contain information about which individual computational result is addressed by it. Additionally, a binding needs to include references to the source code lines and data subsets used to produce that result. A further desirable feature for authors and readers is to be able to manipulate the parameters underpinning a result and then to re-compute them using the changed values. To facilitate this, a binding needs to include information about what parameters can be manipulated within what range and a reference to a UI widget that enables users to change the parameter. The description of the dataset finally enables readers to substitute the underlying dataset with their own compatible one.

Publishers and libraries are the second set of stakeholders with needs that impact the design of the bindings. Both maintain infrastructures to host journals or to archive scientific articles and the attached research materials. In order to facilitate the integration of a binding into these infrastructures, it needs to be encoded in a format that is standardised and easily manageable. Encoding bindings as a JSON object which can be part of a paper's metadata or transformed into other formats (e.g. XML) helps to meet these requirements. 
Finally, we also considered software developers who engineer and operate web applications for publishers and libraries. Software developers need to design and implement the UI for authors to create a binding and for readers to use the resulting interactive figure. The implementation of a binding does not require a specific technology (e.g. Plumber) and thus could be different from what we suggest in this work. However, the results should be based on open data formats (e.g. GeoJSON, .csv) to avoid the need for licensed software to display and reuse closed data formats.

In the following subsections, we elaborate on the individual components of a binding in detail. Figure 1 shows an example binding containing the elements mentioned above (computational results, code links, UI widgets and references to data).

\subsection{Computational result}

A binding contains a unique reference to the corresponding computational result. This result can be a figure, table, or number in the manuscript, i.e. the source file (e.g. RMarkdown) used to render the PDF or HTML file. In the case of a figure or a table, it is sufficient to include the number (e.g. "Figure 1") which is unique throughout the document and always refers to the same result. Referring to a numeric result in the manuscript is more complex and prone to being ambiguous. For example, the number " 5 " in a text might be a numeric result or a chapter number. For a distinct identification of this result, the binding specifies its location in the article. This requires the file name (e.g. "analysis.Rmd"), the line number in that file, and a short textual paragraph, in which the numeric result occurs only once, as the same number might appear multiple times in the same line.

The most fine-grained binding refers to an individual computational result. However, the same code and data described in a binding might produce several numbers, tables, and figures. In this case, a binding might refer to multiple results. Figure 1 shows an example binding for a computational result of the type "figure" and the actual result "Figure 3". The remaining components are described in the following subsections.

\subsection{Source code}

In order to enable the inspection of source code, a binding specifies which source code lines from which script files are required to re-compute the computational result. To enable one to manipulate the analysis underpinning the result, a binding describes the parameter that should be manipulated, i.e. the name of the parameter, its initial value, and the line number in the corresponding code file. A single binding can contain a set of parameters. The source code lines provided in a binding need to be executable. Otherwise it is not possible to create interactive figures or to easily reuse the code for one's own work. For this reason, the code lines contain the entire analysis pipeline to re-compute the result. This includes the importing of libraries, loading and processing of the data, analysis, and the final output function.

The example binding in Figure 1 shows how the description of the code might look like: It specifies the file "analysis.Rmd" that contains the analysis pipeline for "Figure 3", which source code lines are needed from that file ("20-23", " $45-55$ "), and the parameter that should be manipulated, i.e. the parameter "velocity" with the initial value " 2.0 " in line " 23 " of the file "analysis.Rmd". This set of information allows for extracting the required code lines into an executable file which only produces the specified result. This file can then be executed with the new parameter value or reused by readers for their own work.

\subsection{UI widget}

UI widgets can be used to manipulate the parameter values specified in the source code component. The choice of the UI widget depends on the data type of the parameter, e.g. numeric, text, boolean, and the number of potential alternatives. While sliders are suitable for manipulating numeric

Proc. ACM Hum.-Comput. Interact., Vol. 3, No. EICS, Article 16. Publication date: June 2019. 


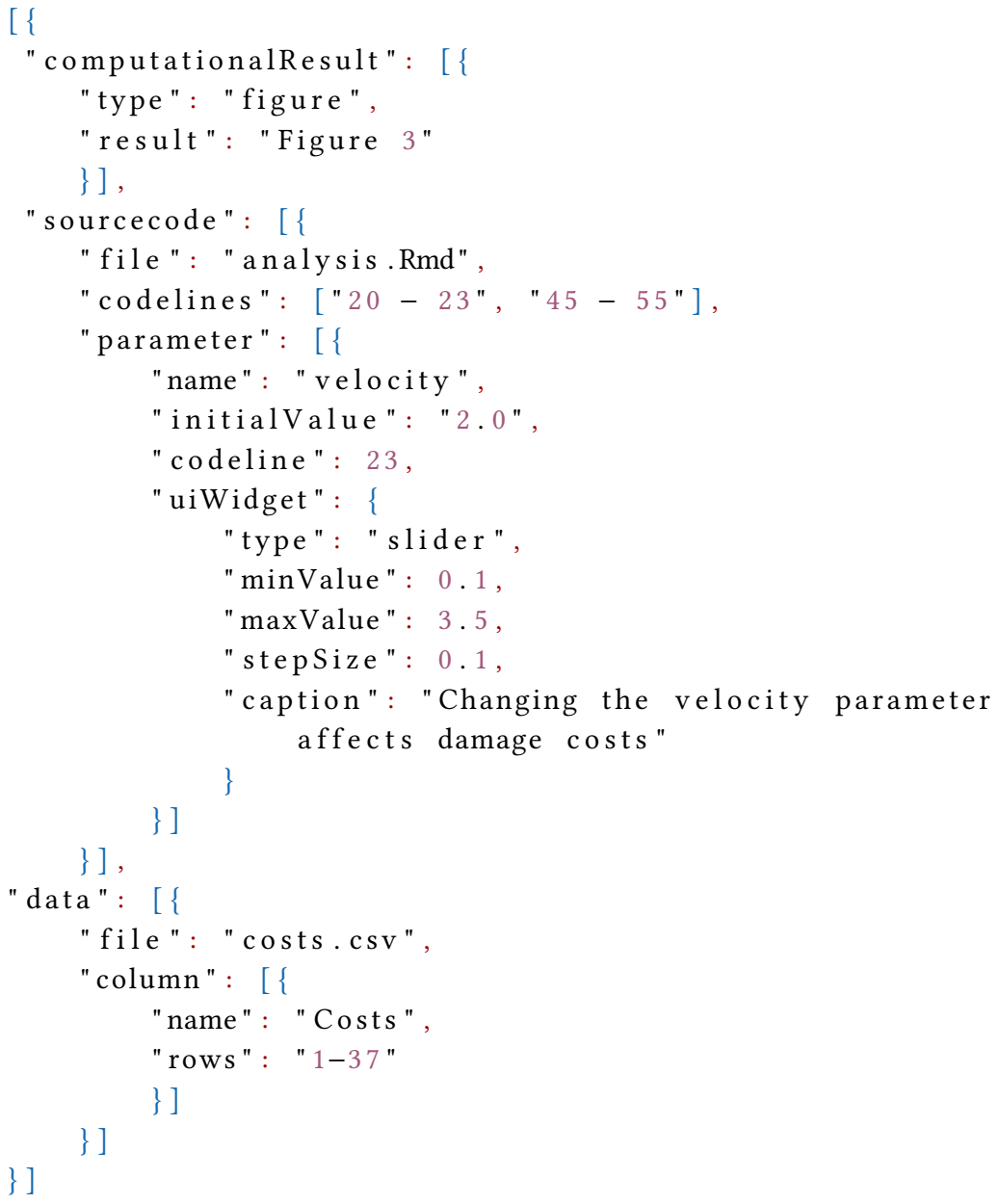

Fig. 1. Example binding stored as JSON object containing the five components computational result, source code, UI widget, and data. The interactive figure that is generated from this binding is shown in Figure 3.

parameters in a certain range (e.g. 1-100), radio buttons are more appropriate for changing textual parameters, such as the model used in a functionality (see e.g. the "vgm" functionality in $R$ and the options, e.g. "gaussian", "matern", "exponential"). A binding requires further information about the selected UI widget: For example, a slider needs a minimum/maximum value and a step size, whereas radio buttons require a set of potential alternatives to the initial value. To enable independent manipulation of different parameters in the same binding, one UI widget corresponds to one parameter. UI widgets only manipulate the code underlying a figure and not the dataset, since all computational steps related to data processing (deleting outliers, transformation) should be scripted in the code. Manual data processing should be avoided for the sake of reproducibility [41].

The binding instance (Figure 1) connects a slider to the parameter "velocity". The range of the slider is described by the minimum value " 0.1 ", maximum value " 3.5 ", and the step size " 0.1 ". Finally, authors can add a caption to explain the purpose of the slider. 


\subsection{Data}

Usually, the source code runs the analysis on data. A binding specifies which data subsets are needed to re-compute an individual result. To achieve this purpose, a binding contains at least the name of the data file. For papers that are based on results that load data from a library, instead of the file name, the binding can then specify which function in the code loads the data. In addition, authors can specify single table columns and rows. This fine-grained information is not required for the execution of the source code. However, a key benefit of a binding is the explicit description of how a certain result was achieved. A further benefit relates to being able to substitute the data underlying the result. In creating their own data to substitute for the original one, readers can avoid common interoperability issues, e.g. with respect to data formats, column names, and data types.

The data component is also included in the example binding (Figure 1). The computational result is based on the rows " $1-37$ " in the column "Costs" of the data file "costs.csv". The readers consequently know which data format to use, how to name the columns, and how many rows the table should have.

\section{REALISATION}

In order to demonstrate the technical realisability of a binding, we implemented a prototypical tool for authors to create a binding, and for readers to use the resulting interactive figure while studying a paper. A video showing all steps is available under https://osf.io/9hb5p/?view_only= b39a75f5c6394c848907f2c49278243c (anonymised for peer review).

\subsection{Author's perspective: Creating a binding}

Figure 2 shows the UI for authors to create a binding. The example illustrates the creation process based on the paper by Dottori et al. [10]. The paper contains a reproducible figure which calculates flood damages based on several parameters, such as flood velocity and duration. According to the authors, changes to the velocity parameter affect the damage calculation. It is thus a suitable candidate for an interactive figure whereby the initial value set by the author can be manipulated. Five steps are required to create the binding as formalised in Figure 1.

Step 1: The author specifies the computational result that should be addressed by the binding.

Step 2: Then, the author marks only those source code lines that are required to produce the corresponding result including the import of libraries and data, data processing, analysis, and the output function (see Figure 2, top).

Step 3: Next, the author selects the parameter that should be manipulated by marking it in the code subset (Figure 2, bottom). The selected parameter and its initial value are shown on the right.

Step 4: Afterwards, the author configures the UI widget. Figure 2 (bottom) exemplifies the configuration of a slider having a minimum value, maximum value, and a step size. Additionally, the author can add a caption to explain the interactive figure.

Step 5: In a final step, the author indicates which data subset is required to produce the specified result, i.e. the file name, columns, and rows.

\subsection{Reader's perspective: Using the interactive figure}

Figure 3 illustrates the UI for readers, including the interactive figure based on the binding provided by the author. Readers can study the scientific article on the left side of the UI. The computational results for which a binding exists are highlighted in bold. If readers would like to quickly manipulate the original parameter mentioned in the caption of the figure (blue box), they can use the interactive figure on the right side of the UI. Readers can change the parameter "velocity" in order to see how different values affect the damage calculation. A short description at the top of the interactive 

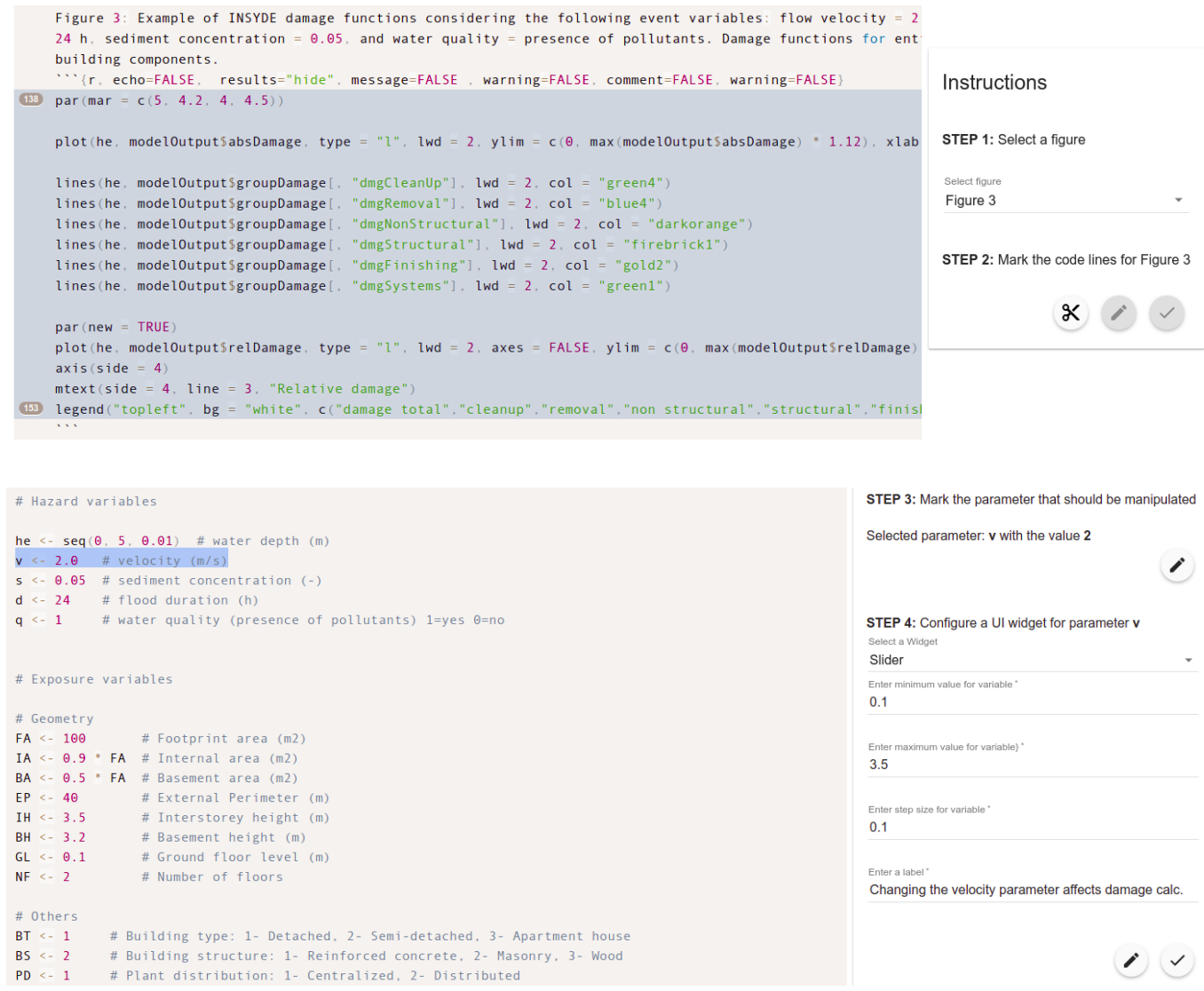

Fig. 2. Creating a binding in five steps. Top: Selecting the computational result and the source code lines which produce it (Step 1 and 2). Bottom: Specifying the parameter that should be manipulated and configuring the UI widget (Step 3 and 4). In the fifth step (not shown), authors specify the data subset (see video).

figure indicates the parameter that can be manipulated, its initial value (green box), and the range of the slider. Below, readers can use the slider. The new parameter value is visible on the right of the slider (black box). The figure adapts each time the reader changes the parameter value. The reader can then compare the original with the manipulated visualisation. In addition, readers can inspect the code and the data underlying the particular figure by pressing the button "Inspect code and data" below the figure.

\subsection{Technical details}

We built the tools on top of the open source web application presented in [27]. The implementation of the frontend tool ${ }^{3}$ is based on AngularfS ${ }^{4}$ (a JavaScript framework) and MaterialUI ${ }^{5}$ (a UI framework). For the backend service ${ }^{6}$ we used node.js ${ }^{7}$ (a server-sided platform for creating web

\footnotetext{
${ }^{3}$ URL to GitHub will be provided after peer review

${ }^{4}$ AngularJS: https://angularjs.org/, last access of this and the following URLs: 21.02.2019.

${ }^{5}$ MaterialUI: https://material.angularjs.org/latest/

${ }^{6} \mathrm{URL}$ to GitHub will be provided after peer review

${ }^{7}$ Node: https://nodejs.org/en/
} 

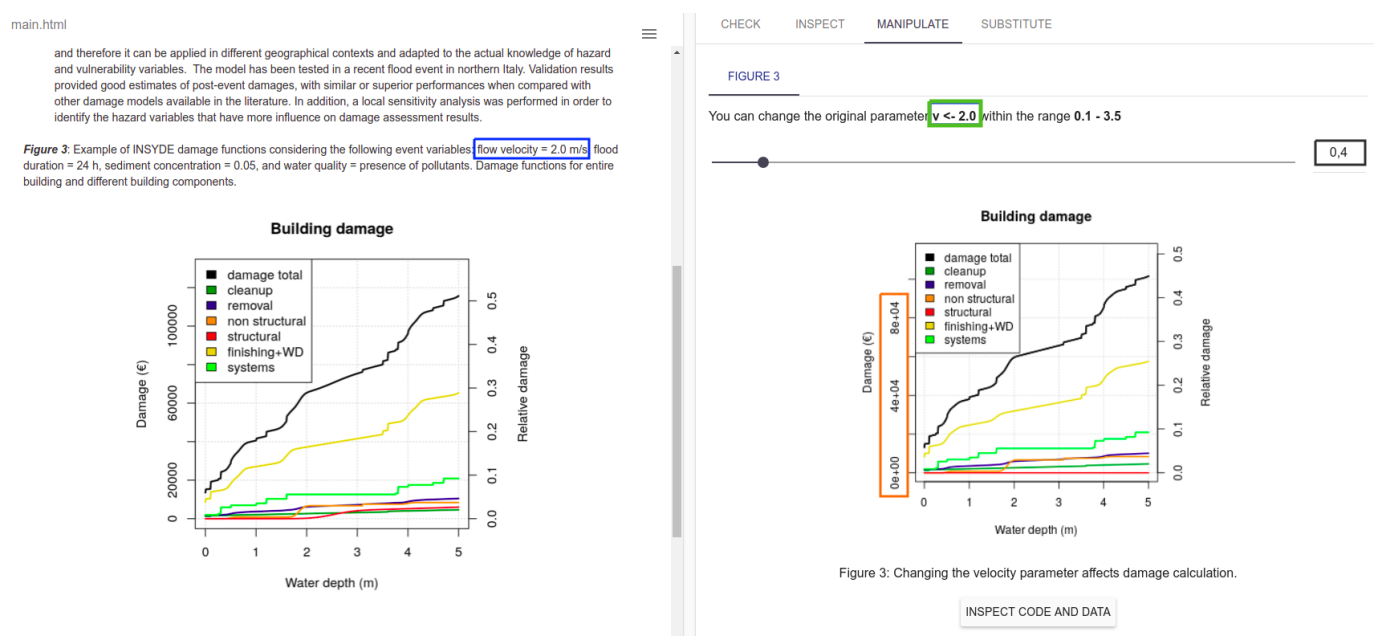

Fig. 3. In parallel to reading the actual article on the left, readers can also change the original parameter (blue box: "velocity $=2.0$ ") by using the slider on the right and see how the new value (black box: "0.4") affects the output (orange box: "damage costs").

servers), $\operatorname{Docker}^{8}$ (a software for encapsulating applications), and Plumber ${ }^{9}$ (an $R$ package for transforming $R$ code into a web api).

\section{EVALUATION}

In order to gain initial insights into the applicability of bindings and the effort required to create them, we produced interactive figures from existing scientific papers. The materials underlying the results in this chapter are accessible as an Open Science Framework project: https://osf.io/9hb5p/ (anonymised for peer review).

\subsection{Materials}

We used a subset of the paper corpus described in the aforementioned reproducibility study [28]. The 41 papers contained therein are all open access, and they come with code and data attached. From these, we excluded 9 papers since we were not able to execute the source code. Additionally, we excluded 8 papers, because they did not produce figures which were included in the original paper. For practical reasons, we did not consider one paper which had an execution time longer than one hour ${ }^{10}$. Overall, we included 23 papers in our evaluation; 15 of these come from the geosciences and were published by Copernicus Publications ${ }^{11}$ and the remaining 8 describe statistical software for the $R$ programming environment and were published in the fournal of Statistical Software ${ }^{12}$.

\subsection{Procedure}

Our first goal was to obtain insights into the applicability of bindings to existing scientific articles. For each paper, we first identified those source code lines that were used to produce an individual

\footnotetext{
${ }^{8}$ Docker: https://www.docker.com/, last access for this and the following URL: 21.02.2019.

${ }^{9}$ Plumber: https://www.rplumber.io/

${ }^{10}$ Laptop: Dell, Ubuntu 16.04 LTS 64 Bit, Intel® ${ }^{\circ}$ ore $^{\mathrm{TM}} \mathrm{i} 7-6500 \mathrm{U}$ CPU @ 2.50GHz x 4, 15,6 GiB RAM.

${ }^{11}$ Copernicus Publications: https://publications.copernicus.org/, last access for this and the following URLs: 21.02.2019.

${ }^{12}$ Journal of Statistical Software: https://www.jstatsoft.org/index
} 
figure in the paper. For this purpose, we searched for the plot function which created the figure and then backtracked the source code until we identified only those code lines that are required to produce the particular figure. We thus reduced the entire source code to a smaller but executable code subset, including the import of libraries and data, data processing, analysis, and the visualisation of the results. To find out how to display the data employed in the analysis, we also noted the file formats, which indicate the structure of the data. In order to create interactive figures, we then searched for parameters which can be manipulated, for example, using a slider or radio buttons. We focused on parameters which influence the results shown in the figure, e.g. the numbers, curves, or statistics, and not the design, e.g. the colour. These parameters and the alternative values should be selected carefully, as changes to the initial configuration might not be meaningful. Consequently, we determined the parameters and the alternative values as follows: First, we scanned the paper and the source code in order to find paragraphs or code comments which discuss parameters used in the code and potential alternatives to their initial configuration. If we were not able to find potential candidates, we examined the functionalities used in the code and consulted the corresponding $R$ documentation ${ }^{13}$ to find parameters and alternative options for the initial values. If this was again unsuccessful, we tried to find alternative parameter values by looking into the used dataset. If this was also not successful, we considered the figure as not being suitable for manipulations and skipped it. Once we found one suitable parameter and at least one potential alternative, we continued with the next figure in the paper. Afterwards, we created Executable Research Compendia (ERCs) [32] out of the paper and the materials. First, we encapsulated the materials into a folder and then uploaded it to a web application ${ }^{14}$ that is capable of handling ERCs. Afterwards, we used the supportive interface for authors (see above) to create a binding, i.e. we extracted the relevant code lines, selected the parameter that should be manipulated, and configured the UI widget. We skipped the step to specify the dataset, as this information is not needed to re-compute the analysis. Finally, we checked if the creation of the interactive figure based on the binding was successful by using the tool for readers (see above).

Our second goal was to investigate the effort required to create and use bindings. For this purpose, we counted the source code lines (excluding comment and empty lines) and connected code blocks in each binding that we created. A connected code block is a sequence of required source code lines (e.g. a functionality) which can be marked at once until an irrelevant code line emerges. These two measures provide initial insights into how many clicks an author has to perform to create the code subset. From these insights, we could also infer which next steps are needed to improve the implementation. In addition, we measured the execution time for each figure to see how long readers have to wait to see the output. A similar performance test was done by Pham et al. [36] who observed faster execution times if only parts of the analysis were re-executed. In order to measure the execution time, we set a time stamp at the beginning and at the end of the analysis. Changes to the parameters might result in very different execution times. As measuring the time for each potential alternative was not realistic, we executed the code with the original parameter value to receive an initial estimate.

\subsection{Results}

5.3.1 Parameters. We excluded 3 papers, since they did not contain figures based on parameters. From the remaining 20 articles, we excluded 30 figures which were not based on parameters and 4 figures which required extensive changes to the original source code to access them individually. In total, we created 83 interactive figures. Figures composed of sub-figures (e.g. "Figure a", "Figure

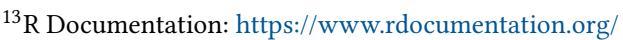

${ }^{14}$ Details left out for anonymisation
} 
b") were counted separately. For these figures, we identified 40 different parameters which can be manipulated. Out of the 40 parameters, we identified 30 by scanning the paper, 5 by reading the corresponding $R$ documentation of the functionality used in the code, 3 by our own examinations, and 2 by comments in the source code made by the original author. For the 40 parameters, we identified alternative values by studying the paper (in 18 cases), by reading the corresponding $R$ documentation (8), by looking into the dataset (8), and from the comments in the code (4). Two parameters were confidence intervals and thus had inherent alternative values, i.e. values between 0 and 1 . We distinguish the following three parameter types: Assumption parameters are values that describe the initial configuration of the analysis, e.g. the velocity of the water in a flooding scenario. These parameters are set by the analyst. Out of the 40 parameters, 16 were categorised as assumption parameters. Method parameters specify a method used as part of a functionality, such as the model used to compute a variogram ${ }^{15}$ (e.g. exponential, gaussian). These parameters have predefined values described, for example, in the $R$ documentation of the corresponding function. We identified 15 parameters as method parameters. Three of these changed the visualisation of the figure, e.g. from a line to a point diagram. Filter parameters specify the data subset used for the analysis, such as a specific column or values measured within a certain period. Changing these parameters consequently results in running the analysis on a different data subset. We found 9 filter parameters. From the 40 parameters, 21 were textual parameters or, in four cases, only had few numeric alternatives. We thus selected radio buttons to manipulate these parameters (a drop-down list would have been possible, too). The remaining 19 parameters could be changed within a certain range. We thus selected a slider to manipulate these parameters.

5.3.2 Execution times. On average, the execution time of the entire analysis took 130 seconds for the first run (median $\tilde{x}=25$ secs., standard deviation $\sigma=241$ secs., values ranged from 1-910 secs.). The second run, i.e. with some parts of the code potentially being cached, took 123 secs. on average (median $\tilde{x}=18.50$ secs., standard deviation $\sigma=232$ secs., values ranged from 1-908 secs.). On average, the analysis of a single figure took 35 seconds (median $\tilde{x}=5$ secs., standard deviation $\sigma=117$ secs., values ranged from $1-878$ secs.) for the first run, and 33 seconds (median $\tilde{x}=3$ secs., standard deviation $\sigma=115$ secs., values ranged from 1-881 secs.) for the second run.

5.3.3 Effort for authors. In order to create figures that can be manipulated using a slider or radio buttons, we had to make a few changes to the original source code: In the code of 41 out of 83 figures, we had to wrap the output function into a generic plot function (i.e. "plot()" in $R$ ), because our software was not able to handle the diversity of existing plot functions. In the code of 33 figures, we had to put the parameter value into a variable. In the original code, the value was passed directly to the functionality. The step was needed to make the parameter and the value uniquely referable and explicitly available in the binding. Having only the value might lead to ambiguous references, for example, if the same value was used multiple times. In one paper, the code of 9 figures was based on a variable which we had to make globally accessible. Finally, in the code of 2 figures, we had to transform the parameter into another data type. In the code of 35 figures, no changes were required. On average, the entire code underlying a paper had 336 lines of code (median $\tilde{x}=135$, standard deviation $\sigma=529$, values ranged from 36-2199). The code of a single figure was composed of 245 lines of code on average (median $\tilde{x}=28$, standard deviation $\sigma=545$, values ranged from 4-1938). This means that, in the worst case, authors need to mark 245 single source code lines. However, usually several lines of code can be summarised to a connected code block, i.e. continuously connected lines of code, such as a function, resulting in a lower number of clicks. On average, a binding had 9 code blocks (median $\tilde{x}=7$, standard deviation $\sigma=8$, values ranged from 1-35). This means that

$\overline{{ }^{15} \mathrm{vgm} \text { function }}$ in $R$ : https://www.rdocumentation.org/packages/gstat/versions/1.1-6/topics/vgm, last access: 21.02.2019. 
authors have to mark 9 code blocks on average to create a binding. However, the high standard deviations, particularly regarding the number of code lines, show that the numbers are only rough estimates.

5.3.4 Data. The data formats used for the analyses underlying the results are diverse. From the 23 articles, 15 included datasets that were stored in open data formats, i.e. $\operatorname{csv}$ (9 papers), txt (8), $14 c$ (1), asc (1), and dat (1). These files can be inspected by users without additional software. In 9 articles, only open formats were used for the analysis. Out of the 23 articles, 14 contained binary files, i.e. Rdata (4), $r d s$ (2), shapefile (2), and sav (1). In addition, 7 datasets were loaded from an $R$ package by calling the name of the dataset in the code. It is not possible to indicate datasets coming from a package the way we did in the example binding above. Instead of the data file, the data component in a binding specifies the name of the dataset and, if applicable, the columns and rows. In case of binary files, readers need additional software to see the content. In 8 papers, only binary files were used for the analysis underlying the results.

\section{DISCUSSION}

The results of the evaluation provide initial evidence that bindings are technically feasible and can be applied to a range of scientific articles. From our sample composed of 23 papers, 20 included figures based on parameters that can be manipulated with the help of UI widgets. The 20 articles included 30 figures which did not have parameters suitable for manipulations. However, in total, we found and successfully implemented 83 interactive figures which can be manipulated using a slider or radio buttons.

\subsection{Parameters}

It is crucial to select the parameters carefully. We tried to avoid meaningless manipulations by studying the paper, comments in the code, and the $R$ documentation. From the 40 parameters, we selected 37 based on these three sources. From these 37 cases, the original author discussed the parameter in the paper or in the code in 32 cases, and suggested alternative values in 22 cases. These parameters are probably the most suitable candidates for manipulations and show that there might be some need for interactive figures. Nevertheless, consulting the documentation of a certain functionality might result in parameters that support the understanding of how a function works, for example, as part of a teaching class. Since we were not the authors of the selected papers, identifying parameters and value ranges required some effort on our part, which would not be the case if authors (as domain experts) picked the parameters themselves.

We subdivided the parameters into three categories: Assumption parameters were "invented" by the original author, for example, as part of a model. Reviewers and readers might ask about the role of these parameters in the model and how it affects the final result. In contrast, method parameters are predefined, for example, in the corresponding software documentation. The question arises why a certain initialisation was selected and how alternative values change the output. Finally, filter parameters specify the data subset used for the analysis and were also defined by the author. Readers and reviewers should know about these parameters to assess whether the overall model is valid. Bindings can help to answer these questions about parameters, for example, by providing means to manipulate them. The 40 parameters can be manipulated using a slider or radio buttons. In practice, the choice for a suitable UI widget would be made by the original author which might be different from what we have selected. 


\subsection{Effort and user interface}

The UI showed the process of creating and using a binding, and also delivered initial insights into the required effort. Fortunately, only few changes to the original source code were needed, such as wrapping a specific output functionality into a more generic one. This might be a reasonable amount of work for authors to make their source code ready for creating interactive figures based on bindings. On average, we had to extract 245 lines of code in 9 code blocks. This number is not high but might constitute a burden for authors to invest time. A (semi-)automatic code extraction might be one way to overcome this barrier. In addition, if authors were aware of the requirements for creating bindings, they could incorporate these into their code from the beginning rather than retrospectively modifying finished code. Beyond the effort to select code lines, only a few clicks are required to specify a UI widget.

The execution times of the interactive figures provide initial insights into how long readers have to wait until they see the output. This is important because each manipulation requires executing the entire code with the new parameter. Half of the 83 figures took not more than 5 seconds, whereas nine took longer than 60 seconds. Manipulations to the parameters thus rarely led to immediate changes in the figure, which might go against users' expectations. Hence, showing a progress bar, pre-rendering outputs, or catching intermediate results might be needed to avoid long waiting times for readers. While checking the interactive figures using the UI for readers, we noticed that changes in the figure after manipulating the parameter might be difficult to spot. For example, curves in a line diagram only varied moderately. For this reason, readers would benefit from a comparison tool which highlights the differences between the original and the manipulated figure, e.g. with the help of change detection algorithms [39].

\subsection{Data}

The data used for the analysis might pose a problem. Open data formats, e.g. csv and geofSON, can be easily displayed in a web application and are readable by humans and machines. In contrast, binary data formats, such as RData or shapefiles, require additional software to make them accessible for users. So far, the implementation does not work with binary data formats and thus might incentivise authors to make use of open formats. In addition, this issue affects the substitution of the dataset underlying an individual computational result. It becomes more challenging for readers to create their own interoperable dataset if the original dataset was stored in a binary file format. However, even in open data formats, the structure of tables (e.g. column names, data types) varies considerably, making it difficult to achieve interoperability between datasets [27]. By specifying the data subset, a binding might support readers in completing this task.

\subsection{Benefits}

Bindings are not a necessity for manipulating a parameter in the source code. In theory, an interested reader could take the code, run it locally, and adjust the parameters. However, UI widgets make it much easier to successfully complete this task and allow one to quickly check, for example, how the results change if the underlying assumptions change. This could be the task of reviewers who would like to verify the reported results and to challenge the assumptions made by the author prior to suggesting publication of the paper [36]. In addition, readers do not have to search for the parameter and to set up the analysis in their own local environment. Instead, they can use the interactive figure in parallel to studying the paper. Moreover, the fine-grained links between the three components code, data, and a specific result explicitly refer to single parameters which affect the output. This might help to make computational results more transparent. A further benefit of a binding is that it results in executable source code. Instead of writing source code from scratch, 
readers can reuse the analysis underlying a specific result and build their research on top of it. While this approach is thus beneficial for readers, it requires a way for others to cite the reused code and data [46]. Bindings can also contribute to searching for papers in a more fine-grained way. For example, bindings might facilitate finding papers that include results based on code using a certain library.

\subsection{Limitations}

The work presented in this paper is subject to a number of limitations. The sample used for the evaluation is small and thus not representative. In addition, we only examined papers with code written in $R$. Further articles from other disciplines based on other programming languages are required to draw more informed conclusions. Nevertheless, the dataset included a variety of figures, which provides an initial evaluation of our approach.

It remains unclear if all parameter manipulations that we applied to the code were meaningful. In practice, parameter and value selection should be done by the original author who knows best which parameters can be manipulated in which way. Nevertheless, most of the parameters and alternative values were chosen because they were mentioned in the paper, in the code, or in the $R$ documentation of the corresponding function.

Manipulating parameters is not relevant for all figures based on computational analyses. In our sample, a couple of figures did not have suitable parameters. However, inspecting the underlying code and data, or substituting the data might still be interesting for readers.

The results regarding the effort to create bindings are only rough measures at this point. For example, the creation of bindings was done by the same person who implemented the prototype and not by the paper authors. In addition, although the number of clicks needed to create an interactive figure is a factual and objective measure, the cognitive effort is also a crucial aspect for the evaluation of the system. To address these two issues, a user study with actual authors might reveal insights regarding the cognitive effort and further issues while using the system, such as the need for more or less time for creating interactive figures. However, such a study should consider that measuring the cognitive workload strongly depends on the paper topic, complexity of the code, as well as the researcher's familiarity with programming and data schemata.

A further crucial aspect is the knowledge the authors need to create interactive papers. The authors mainly need knowledge about their own code in order to select the right and complete set of source code lines, e.g. the functions and parameters that produce a particular result. In our evaluation, we did not consider the time required to spot the source code lines while creating a binding. This was less of an issue for us, because we knew the code in detail, and this might also be true for authors who recently finished the analysis. However, the more time that has passed between finishing the analysis and creating a binding, the more time authors might need to again become familiar with their source code. Nevertheless, even if the authors know their code thoroughly, different issues might occur. For example, they might miss selecting a source code line, function, parameter, or a library resulting in a binding that is not executable. The semi-automatic code extraction mentioned above might help authors to avoid such issues. Ideally, both bindings and the code would be created in tandem while authoring a paper. In addition, we did not discuss the fifth step to create a binding in depth, i.e. specifying the dataset. We skipped this step, because it is not needed to create an interactive figure which allows for manipulating a parameter. The measures indicating the effort for authors are thus not complete. Hence, further research is needed regarding how much time authors need and if they are willing to invest it to create an interactive figure.

One of the key motivations of this work on interactive papers is the possibility to gain an increased understanding. Although some studies and related approaches provided initial evidence 
that interactive papers are better understandable and more transparent (see, for example, the work by Adnan et al. [1], Dragicevic et al. [11], and Wacharamanotham et al. [50]), it remains open to which extent readers obtain additional knowledge compared to 'classic' papers, such as PDFs. Further studies with authors and readers are required to evaluate the bindings concept, for example, in comparison to the existing approaches presented in Related Work. It might be interesting also to consider the invested time. Ideally, readers receive more insights while keeping reading times low. Otherwise, readers might become reluctant to use interactive papers.

In order to realise bindings, the computational results reported in an article need to be reproducible, which is not yet common practice [34]. However, the opportunity to create interactive figures easily based on bindings might incentivise authors to publish open reproducible research.

\section{CONCLUSION}

In this work, we introduced an approach to create interactive papers which support readers and reviewers in inspecting code and data, manipulating parameters, and substituting the dataset underlying the results in a paper. We proposed the concept of a binding based on the needs of stakeholders, such as scientists and publishers. A key benefit of a binding is that it connects the text with the code and the data at a fine-grained level, which in turn not only simplify the (manual) inspection of all components but also enables interactive figures. While studying an article, readers and reviewers can use these interactive figures to understand how a specific result was computed and quickly check, how changes to the parameters affect the result. In addition, a binding might foster the reuse of existing code, resulting in more citations for the original author and less work for the reader. This paper thus makes three key contributions: (1) We first presented the concept of a binding. A binding breaks the coarse-grained linkage between the paper, data, and code in order to create fine-grained links between those source code lines and data subsets that are needed to produce an individual computational result (e.g. a figure). On top of that, a binding explicitly specifies the parameters underlying the results and the UI widgets that can be used to interactively manipulate the parameter values defined by the author. (2) Next, we demonstrated the technical feasibility of the concept by implementing a prototypical toolkit which supports creation and usage of bindings. While the implementation showed that only a few steps are needed to create an interactive figure, authors still need detailed knowledge of their code. (3) Finally, we evaluated the approach by applying the binding concept and its implementation to existing scientific papers. We successfully created 83 interactive figures from 20 scientific articles which allow readers and reviewers to manipulate a parameter underpinning the results shown in a figure. In addition, we also identified a number of potential issues, such as long computation times for some figures, and the effort needed to select the relevant code lines. Despite these issues, a key benefit of bindings is that they make it easier for readers and reviewers to explore how an individual result, e.g. a specific figure, was computed, which parameters were used, and how changes to these affect the outcome.

\section{FUTURE WORK}

The next step is to conduct user studies with authors and readers of scientific articles to collect insights regarding usability and user experience. For this reason, we will improve the current implementation to enable the manipulation of more than one parameter per figure by using different UI widgets. To support the selection of relevant code lines, we will implement a backtrack feature which only requires selecting the functionality that creates the output and not the entire analysis pipeline. We will also implement tools for readers for identifying differences between the original and the manipulated figure, e.g. by using an overlay of both figures or a side-by-side comparison. Based on these advancements, we are also planning to run a study to assess the detailed impact of using interactive papers on readers' understanding compared to traditional publications.

Proc. ACM Hum.-Comput. Interact., Vol. 3, No. EICS, Article 16. Publication date: June 2019. 


\section{REFERENCES}

[1] Muhammad Adnan, Mike Just, and Lynne Baillie. 2016. Investigating Time Series Visualisations to Improve the User Experience. In Proceedings of the 2016 CHI Conference on Human Factors in Computing Systems (CHI '16). ACM, 5444-5455.

[2] JJ Allaire, Yihui Xie, Jonathan McPherson, Javier Luraschi, Kevin Ushey, Aron Atkins, Hadley Wickham, Joe Cheng, and Winston Chang. 2018. rmarkdown: Dynamic Documents for $R$. https://CRAN.R-project.org/package=rmarkdown R package version 1.10. Accessed: 2019-02-21.

[3] Giuliano Antoniol, Gerardo Canfora, Gerardo Casazza, Andrea De Lucia, and Ettore Merlo. 2002. Recovering traceability links between code and documentation. IEEE transactions on software engineering 28, 10 (2002), 970-983.

[4] Alberto Bacchelli, Michele Lanza, and Romain Robbes. 2010. Linking e-mails and source code artifacts. In Proceedings of the 32nd ACM/IEEE International Conference on Software Engineering-Volume 1. ACM, 375-384.

[5] Nick Barnes. 2010. Publish your computer code: it is good enough. Nature News 467, 7317 (2010), 753-753.

[6] Winston Chang, Joe Cheng, JJ Allaire, Yihui Xie, and Jonathan McPherson. 2018. shiny: Web Application Framework for $R$. https:/CRAN.R-project.org/package=shiny R package version 1.1.0. Accessed: 2019-02-21.

[7] Fernando Chirigati, Rémi Rampin, Dennis Shasha, and Juliana Freire. 2016. Reprozip: Computational reproducibility with ease. In Proceedings of the 2016 International Conference on Management of Data. ACM, 2085-2088.

[8] Julien Colomb and Björn Brembs. 2014. Sub-strains of Drosophila Canton-S differ markedly in their locomotor behavior. F1000Research 3 (2014).

[9] Benjamin M. Delory, Mao Li, Christopher N. Topp, and Guillaume Lobet. 2018. archiDART v3. 0: A new data analysis pipeline allowing the topological analysis of plant root systems. F1000Research 7 (2018).

[10] Francesco Dottori, Rui Figueiredo, Mario LV Martina, Daniela Molinari, A. Scorzini, et al. 2016. INSYDE: a synthetic, probabilistic flood damage model based on explicit cost analysis. Natural Hazards and Earth System Sciences 16 (2016), 2577-2591.

[11] Pierre Dragicevic, Yvonne Jansen, Abhraneel Sarma, Matthew Kay, and Fanny Chevalier. 2019. Increasing the Transparency of Research Papers with Explorable Multiverse Analyses. In CHI 2019 - The ACM CHI Conference on Human Factors in Computing Systems. ACM.

[12] Bastian Drees, Angelina Kraft, and Thomas Koprucki. 2018. Reproducible and comprehensible research results through persistently linked and visualized numerical simulation data. Optical and Quantum Electronics 50, 2 (2018), 59.

[13] Steve M Easterbrook. 2014. Open code for open science? Nature Geoscience 7, 11 (2014), 779.

[14] Florian Echtler and Maximilian Häußler. 2018. Open Source, Open Science, and the Replication Crisis in HCI. In Extended Abstracts of the 2018 CHI Conference on Human Factors in Computing Systems. ACM, alt02.

[15] Gabriel Fils, Zhihao Yuan, Tanu Malik, et al. 2017. Sciunits: Reusable Research Objects. In e-Science (e-Science), 2017 IEEE 13th International Conference on. IEEE, 374-383.

[16] Robert Gentleman and Duncan Temple Lang. 2007. Statistical analyses and reproducible research. Fournal of Computational and Graphical Statistics 16, 1 (2007), 1-23.

[17] Virginia Gewin. 2016. Data sharing: An open mind on open data. Nature 529, 7584 (2016), 117-119.

[18] Yolanda Gil, Varun Ratnakar, and Daniel Garijo. 2015. OntoSoft: Capturing scientific software metadata. In Proceedings of the 8th International Conference on Knowledge Capture. ACM, 32.

[19] Jeremy Goecks, Anton Nekrutenko, and James Taylor. 2010. Galaxy: a comprehensive approach for supporting accessible, reproducible, and transparent computational research in the life sciences. Genome biology 11, 8 (2010), R86.

[20] Steven N. Goodman, Daniele Fanelli, and John P. A. Ioannidis. 2016. What does research reproducibility mean? Science translational medicine 8, 341 (2016), 341ps12-341ps12.

[21] Miriam Greis, Hendrik Schuff, Marius Kleiner, Niels Henze, and Albrecht Schmidt. 2017. Input Controls for Entering Uncertain Data: Probability Distribution Sliders. Proceedings of the ACM on Human-Computer Interaction 1, 1 (2017), 3.

[22] Stevan Harnad, Tim Brody, Francois Vallieres, Les Carr, Steve Hitchcock, Yves Gingras, Charles Oppenheim, Heinrich Stamerjohanns, and Eberhard R. Hilf. 2004. The access/impact problem and the green and gold roads to open access. Serials review 30, 4 (2004), 310-314.

[23] Anett Hoppe, Jascha Hagen, Helge Holzmann, Günter Kniesel, and Ralph Ewerth. 2018. An Analytics Tool for Exploring Scientific Software and Related Publications. In Digital Libraries for Open Knowledge, Eva Méndez, Fabio Crestani, Cristina Ribeiro, Gabriel David, and João Correia Lopes (Eds.). Springer International Publishing, Cham, 299-303.

[24] Tomi Kauppinen and Giovana Mira de Espindola. 2011. Linked Open Science-Communicating, Sharing and Evaluating Data, Methods and Results for Executable Papers.. In ICCS. 726-731.

[25] Thomas Kluyver, Benjamin Ragan-Kelley, Fernando Pérez, Brian E. Granger, Matthias Bussonnier, Jonathan Frederic, Kyle Kelley, Jessica B. Hamrick, Jason Grout, Sylvain Corlay, et al. 2016. Jupyter Notebooks-a publishing format for reproducible computational workflows.. In ELPUB. 87-90.

[26] Donald Ervin Knuth. 1984. Literate programming. Comput. f. 27, 2 (1984), 97-111. 
[27] Markus Konkol and Christian Kray. 2018. In-depth examination of spatio-temporal figures in open reproducible research. Cartography and Geographic Information Science (2018).

[28] Markus Konkol, Christian Kray, and Max Pfeiffer. 2018. Computational reproducibility in geoscientific papers: Insights from a series of studies with geoscientists and a reproduction study. International fournal of Geographical Information Science (2018), 1-22.

[29] Friedrich Leisch. 2002. Sweave: Dynamic generation of statistical reports using literate data analysis. In Compstat. Springer, $575-580$.

[30] Jon Mease. 2018. Bringing ipywidgets Support to plotly. py. (2018).

[31] Daniel Nüst, Carlos Granell, Barbara Hofer, Markus Konkol, Frank O. Ostermann, Rusne Sileryte, and Valentina Cerutti. 2018. Reproducible research and GIScience: an evaluation using AGILE conference papers. Peerf 6 (2018), e5072.

[32] Daniel Nüst, Markus Konkol, Edzer Pebesma, Christian Kray, Marc Schutzeichel, Holger Przibytzin, and Jörg Lorenz. 2017. Opening the publication process with executable research compendia. D-Lib Magazine 23, 1/2 (2017).

[33] Jeroen Ooms. 2018. opencpu. https://CRAN.R-project.org/package=opencpu Accessed: 2019-02-21.

[34] Frank O. Ostermann and Carlos Granell. 2017. Advancing science with VGI: Reproducibility and replicability of recent studies using VGI. Transactions in GIS 21, 2 (2017), 224-237.

[35] Jeffrey M. Perkel. 2018. Data visualization tools drive interactivity and reproducibility in online publishing. Nature 554, 7690 (2018), 133-134.

[36] Quan Pham, Tanu Malik, and Ian Foster. 2013. Using Provenance for Repeatability. TaPP 13 (2013), 2.

[37] Quan Pham, Tanu Malik, Ian Foster, Roberto Di Lauro, and Raffaele Montella. 2012. SOLE: linking research papers with science objects. In International Provenance and Annotation Workshop. Springer, 203-208.

[38] R Core Team. 2018. R: A Language and Environment for Statistical Computing. R Foundation for Statistical Computing, Vienna, Austria. https://www.R-project.org Accessed: 2019-02-21.

[39] Richard J Radke, Srinivas Andra, Omar Al-Kofahi, and Badrinath Roysam. 2005. Image change detection algorithms: a systematic survey. IEEE transactions on image processing 14, 3 (2005), 294-307.

[40] Thomas Rathfux, Roman Popp, and Hermann Kaindl. 2016. Adding custom widgets to model-driven GUI generation. In Proceedings of the 8th ACM SIGCHI Symposium on Engineering Interactive Computing Systems. ACM, 16-26.

[41] Geir Kjetil Sandve, Anton Nekrutenko, James Taylor, and Eivind Hovig. 2013. Ten simple rules for reproducible computational research. PLoS computational biology 9, 10 (2013), e1003285.

[42] Carson Sievert, Chris Parmer, Toby Hocking, Scott Chamberlain, Karthik Ram, Marianne Corvellec, and Pedro Despouy. 2017. plotly: Create Interactive Web Graphics via 'plotly. js'. R package version 4.7. 1.

[43] Dalmeet Singh Chawla. 2015. 'Living figures' make their debut. Nature News 521, 7550 (2015), 112.

[44] Stefan Steiniger and Erwan Bocher. 2009. An overview on current free and open source desktop GIS developments. International fournal of Geographical Information Science 23, 10 (2009), 1345-1370.

[45] Victoria Stodden, Matthew S. Krafczyk, and Adhithya Bhaskar. 2018. Enabling the Verification of Computational Results: An Empirical Evaluation of Computational Reproducibility. In Proceedings of the First International Workshop on Practical Reproducible Evaluation of Computer Systems. ACM, 3.

[46] Victoria Stodden, Marcia McNutt, David H. Bailey, Ewa Deelman, Yolanda Gil, Brooks Hanson, Michael A. Heroux, John P. A. Ioannidis, and Michela Taufer. 2016. Enhancing reproducibility for computational methods. Science 354, 6317 (2016), 1240-1241.

[47] Trestle Technology, LLC. 2018. plumber: An API Generator for R. https://CRAN.R-project.org/package=plumber R package version 0.4.6. Accessed: 2019-02-21.

[48] Ramnath Vaidyanathan, Yihui Xie, JJ Allaire, Joe Cheng, and Kenton Russell. 2016. htmlwidgets: HTML Widgets for R. https://CRAN.R-project.org/package=htmlwidgets R package version 1.3. Accessed: 2019-02-21.

[49] Bret Victor. 2018. Explorable Explanations. http://worrydream.com/\#!/ExplorableExplanations. Accessed: 2019-02-21.

[50] Chat Wacharamanotham, Krishna Subramanian, Sarah Theres Völkel, and Jan Borchers. 2015. Statsplorer: Guiding novices in statistical analysis. In Proceedings of the 33rd Annual ACM Conference on Human Factors in Computing Systems. ACM, 2693-2702.

[51] L.J. Welty, L.V. Rasmussen, A.S. Baldridge, and E. Whitley. 2016. StatTag. Galter Health Sciences Library, Chicago, Illinois, United States.

[52] Mark D. Wilkinson, Michel Dumontier, IJsbrand Jan Aalbersberg, Gabrielle Appleton, Myles Axton, Arie Baak, Niklas Blomberg, Jan-Willem Boiten, Luiz Bonino da Silva Santos, Philip E. Bourne, et al. 2016. The FAIR Guiding Principles for scientific data management and stewardship. Scientific data 3 (2016).

[53] Kanit Wongsuphasawat, Dominik Moritz, Arvind Satyanarayan, and Jeffrey Heer. 2018. Vega-Lite - A Grammar of Interactive Graphics. https://vega.github.io/vega-lite/. Accessed: 2019-02-21.

Received February 2019; revised March 2019; accepted April 2019 